\title{
Book Recommendation: Advances in Water Quality Control
}

\section{Gail Krantzberg, Aysegul Tanik et al.}

Scientific Research Publishing, 2010

316 pages

ISBN: 978-1-935068-08-2

Paperback (US\$89.00) E-book (US\$89.00)

Order online: www.scirp.org/book

Order by email: bookorder@scirp.org

\section{Background}

Water is for sure the only common and global issue that interests all the living bodies of the world including humans, flora and fauna. Without water, survival is not possible. It is among the main natural resources that recognizes no borders. The two components of water are namely quality and quantity. World water resources are estimated to be 1400 million $\mathrm{km}^{3}$; however, only a minor portion of this potential amount is usable water. As the amount of usable/accessible water differs from country to country, place to place, season to season, water shortage is experienced in some countries. It is well known that water resources are not equally distributed according to population densities among the countries. Some countries have abundant water resources, whereas some others suffer from inadequate water, and even face severe water scarcity problems. Currently, a portion of accessible global water resources are polluted especially through human-induced activities, and can no longer be used. Another portion of water is lost during transmission due to lack of efficient and proper infrastructure. Thus, pollution of water resources leads to serious sanitary problems. $1 / 5^{\text {th }}$ of the world's population that accounts to approximately 1.1 billion people is under the risk of water-related diseases, and moreover each year many people die as they do not have safe drinking water. The main reason of this fact is lack of adequate infrastructure rather than water scarcity. For that reason, one of the Millennium Development Goals, announced at the United Nations General Council in year 2000, is to decrease the number of people that suffer from clean water access by 50\% by year 2015 .

The sectoral distribution of water consumption as a global average is dominated by agricultural and green land irrigation with an overall value of $70 \%$, followed by industrial uses with $22 \%$, and $8 \%$ is shared by domestic uses as referred in the UN World Water Development Report of year 2003. It is for sure that our limited natural water resources are highly used in agricultural activities, and industrial development and population rise in parallel increase the demand for water to be used in these sectors. Thus, we are trying to increase the quantity of accessible clean and secure water. On the other hand, the wastewater arising from the use of water is an even more significant problem when they are discharged into receiving water bodies. To cope with this problem and protect the well-being of our environment, various types of wastewater treatment systems; primary, secondary, tertiary have to be installed for the point sources of pollutants. Technological advances make it possible to implement different types of wastewater systems to minimize their polluting effects and moreover, the most recent reuse technologies enable to establish advanced systems for reusing wastewater. On one hand, humans pollute their environment through their activities and on the other hand, they work hard and spent time and money in installing wastewater treatment plants to protect their environment. The efforts of the humans in keeping their water resources clean usually become insufficient especially in developing and under-developed countries. Among the reasons, one can address the high investment and operational costs of wastewater treatment plants, the institutional and legal constraints faced in the countries, lack of receiving water and discharge standards, and lack of monitoring and control of the already established plants.

\section{Aims and Readers}

In short, maintaining the required quality of water according to the intended use has now become one of the important issues to be tackled with. This book on $A d$ vances in Water Quality Control emerged at this point. There are various books written worldwide on water quality; however, this book aims to put forth and focus on the recent advances of water quality control. A group of international experts have come together to write this book who target to pass recent available knowledge and information to the readers. The readers and beneficiaries varies from academicians, professional engineers and scientists, to undergraduate and graduate engineering 
students from all nations no matter where they come from; either a developed and a developing country.

\section{Introduction of Chapters}

Keeping in mind the philosophy of "utilization and protection balance”, Chapter 1 refers to water quality requirements emphasizing on the factors that affect water quality. The water quality parameters that need to be considered for various common beneficial uses of water worldwide are mentioned, and the authors focused on the water quality criteria, guidelines and standards in use. As is known, standards need to be set by nations based on their intended use of water by taking into account the socio-economical status and political situation. Reuse of water in various sectors has nowadays become an important topic in the field of water use, as this natural resource is becoming more limited. Chapter 2 is on using the treated wastewater in different sectors; mainly in agricultural activities. As referred previously, the share of water dominates in the agricultural sector and we have to find an alternative water resource to reduce the irrigation water demand with clean water. The advances in reuse technologies have made wastewater treatment available for irrigation. Therefore, this chapter, after introducing the various types of reuses reviews the advanced technologies used in implementing and upgrading agricultural reuse systems. The recent experiences of various countries are mentioned. The management of wastewater as a new resource of water is also referred to enlighten the readers on the important items of reusing treated water. Selection of the most effective and proper wastewater treatment is actually the essential part of generating a new water resource as well as protecting the discharge environments. Thus, the fundamental principles of wastewater treatment are focused in Chapter 3. This chapter covers all the acceptable and universally applied wastewater treatment processes and systems. Another aspect is using wastewater in aquaculture. The historical background using wastewater in aquaculture, probable impacts and technical aspects of fish culture is explained in depth in Chapter 4. Beyond presenting the wastewater treatment technologies and reuse of wastewater in various sectors, one gets interested with the use and application of economic instruments, selection between different instruments to cope with the problem of water pollution. This topic is covered in detail in Chapter
5. Moreover, financing and sustaining wastewater management is another issue that needs to be highlighted. Chapter 6 is devoted to this topic of management where "sustainability" is emphasized as the common key word of technical, legislative and supportive aspects of wastewater management.

Within the past few decades, the advances in computer science made available the use of modern information technologies that makes our lives easier. Chapter 7 is spared to the information systems used nowadays in water quality control. Emphasis is given to the implementation of water quality networks and to water quality monitoring technology. After presenting all the important points of water quality control, now we have to establish a framework for water pollution control. This is covered in Chapter 8 where it is referred that identification and determination of polluting sources need to be done on watershed basis. Watershed based studies require the use of certain modern management tools and instruments. The selection and application of an appropriate management plan for water pollution control and management alternatives form the basis of this chapter. There are various conventional and modern tools and techniques used in water quality interpretation. They are explained in Chapter 9 in detail including a case study on application of salient techniques to groundwater quality assessment.

Chapter 10 is on a case study conducted in Canada on revitalization plans and implementation aiming establishment of a successful clean-up strategy. The lessons learnt from the application are also stated. Chapter 11 is again on a case study of a pollution control strategy implemented for determining the characteristics of Polyaluminum Chloride (PAC) that is used as coagulant prepared by baking process. Chapter 12 concludes the book with a look at low-cost wastewater treatment processes. Along with the conventional wastewater treatment processes, the natural biological treatment systems are overviewed in this chapter in detail. This book is designed in a way to overview the recent advances in water quality control and I hope it will serve to advance the field of water quality control and improve the control actions and strategies to be applied by the nations of the world.

Aysegul Tanik

Istanbul, Turkey 